\title{
Measurement-Based 5G Millimeter-Wave Propagation Characterization in Vegetated Suburban Macrocell Environments
}

\author{
Peize Zhang, Student Member, IEEE, Bensheng Yang, Student Member, IEEE, Cheng Yi, \\ Haiming Wang, Member, IEEE, and Xiaohu You, Fellow, IEEE
}

\begin{abstract}
An empirically based analysis of propagation characteristics in two vegetated suburban areas with different types and fractions of vegetation cover in $5 \mathrm{G}$ millimeter-wave bands is presented. A basic distance-dependent path loss model with a Gaussian random variance for shadow fading is utilized in accordance with the maximum-power directional and omnidirectional measurement data, therein exploiting significant path loss exponents in the presence of vegetation. In comparison with the existing ITU-R and 3GPP models, the effect of dense-leaved trees on path loss prediction is similar to that of buildings, whereas these standard models are inapplicable for sparse obstacle-lineof-sight (OLoS) links. Consequently, an azimuth-angle-based path loss characterization is proposed considering the antenna pattern, beam misalignment, and blockage effects. Moreover, several composite and cluster-level small-scale channel parameters, such as the number of clusters, delay spread, and angular spread, are extracted. Analysis of the first-arrival cluster in the OLoS setting reveals that forward scattering through foliage is still dominant and is expected to produce a larger azimuth angular spread of the arrival and compact multipath components in the time domain compared with line-of-sight and reflected clusters. Measurement results improve existing 3GPP channel models for suburban macrocell scenarios in millimeter-wave bands.
\end{abstract}

Index Terms-Millimeter-wave radio propagation, multipath channels, path loss, propagation measurement, suburban environments, vegetation

\section{INTRODUCTION}

D IVERSE data-driven services and new innovative applications in next-generation mobile communication systems (that is, 5G and beyond) require an ultra-high data rate and ultra-low latency to enhance the quality of mobile users' experience in different deployment scenarios [1], [2]. The use of the plentiful spectrum in the millimeter-wave

Manuscript received Aug. 8, 2019; revised Jan. 26, 2020; accepted Feb. 17, 2020. Date of publication ; date of current. This work was supported in part by the National Key R\&D Program of China under Grant 2018YFB1801101, the National Natural Science Foundation of China under Grants 61671145 and 61960206006, and the Key R\&D Program of Jiangsu Province of China under Grant BE2018121. (Corresponding author: Haiming Wang.)

P. Zhang, B. Yang, C. Yi, and H. Wang are with the State Key Laboratory of Millimeter Waves, Southeast University, Nanjing 210096, China and also with the Purple Mountain Laboratories, Nanjing 211111, China (email: pzzhang@seu.edu.cn, yangbensheng@seu.edu.cn, chengyi@ seu.edu.cn, hmwang@seu.edu.cn).

$\mathrm{X}$. You is with the National Mobile Communications Research Laboratory, Southeast University, Nanjing 210096, China and also with the Purple Mountain Laboratories, Nanjing 211111, China (e-mail: xhyu@ seu.edu.cn).

Color versions of one or more of the figures in this paper are available online at http://ieeexplore.iee.org.

Digital Object Identifier
(mmWave) bands is expected to meet the anticipated capacity boost required by the future wireless era [3], [4]. However, the radio propagation characteristics in the mmWave band will significantly differ from what we have understood in conventional microwave bands. Although recent cellular-type channel measurements have confirmed that not dramatically worse coverage radii (up to approximately $200 \mathrm{~m}$ for nonline-of-sight (NLoS) links in dense urban environments [5][8]) can be realized, high-gain horn antennas or beam-steering array antennas are needed to compensate for severe path loss in the mmWave band [9]-[11]. Moreover, mmWave signals are sensitive to blockages in real environments due to their smaller wavelength compared to the dimensions of physical objects. Consequently, it is crucial to analyze blockage effects, such as human body shadowing [12], foliage attenuation [13]-[15], and building penetration [16], [17], in deploying mmWave networks. For these reasons, a thorough understating of the propagation channel in the concerned mmWave bands plays an important role of achieving the optimum design of cellular networks and the cost-effective placement of base stations (BSs).

The channel characteristics of mmWave cellular systems are site-specific because of their vulnerability to propagation effects. As identified in Recommendation ITU-R P.1411 [18], five outdoor physical operating environments and their propagation impairments are illustrated. In urban microcell and macrocell environments (e.g., urban very-high-rise, urban high-rise, and urban low-rise environments), channel characteristics have been widely investigated in the two $5 \mathrm{G}$ mmWave candidate bands (i.e., $28 \mathrm{GHz}$ and $39 \mathrm{GHz}$ ) [5]-[8]. However, there is a dearth of research focusing on the underlying measurement-based characterization of mmWave channels in suburban macrocell environments except for [19]-[21]. To predict transmission losses for suburban line-of-sight (LoS) and NLoS scenarios at $28 \mathrm{GHz}$, directional measured data were collected to fit the close-in (CI) free-space reference distance model and the alpha-beta-gamma (ABG) model, where the antenna pattern was deembedded based on precisely reconstructing the geometric relationship between the antennas [21], [22]. In [20], the preliminary results (e.g., path loss, shadowing and delay spread) for statistically modeling $28 \mathrm{GHz}$ channel in residential suburban cellular scenario were presented based on a double-directional measurement campaign. Apart from path loss models, the angular dispersion at $24 \mathrm{GHz}$ was analyzed based on the direction-scan-sounding (DSS) method, showing 
that substantially more multipath components (MPCs) can be observed in the suburban scenario compared to the urban case [19]. These measurements, however, did not provide a thorough channel characterization of suburban environments across multiple mmWave bands in terms of the path loss model and spatiotemporal statistics. Meanwhile, the building heights in suburban regions are generally less than several stories (typically three), indicating that the BS antenna height will strongly affect mmWave cellular network coverage [23]-[25].

Notice that a distinguishing feature of suburban areas is their high vegetation coverage, which results in lower LoS probability and greater excess loss because of the propagation through trees and foliage (that is, the obstacle-LoS (OLoS) scenario). The theoretical and empirical modeling of the effect of vegetation media at mmWave frequencies has been extensively conducted [15], [26]-[31]. Following the proposed models in Recommendation ITU-R P.833 [32], an exponential function with respect to carrier frequency, vegetation depth, and elevation angle is utilized to model the foliage attenuation when the transmitter (TX) and receiver (RX) are both outside the vegetated area [15], [28], [29]; in addition, the terrestrial model varying with vegetation depth is used for the case in which only one terminal is located within the extensive vegetation [29], [30]. However, there is limited knowledge on the interaction between channel dispersion (e.g., delay spread [33], [34] and angular spread [15]) and scattering phenomena in the presence of vegetation across multiple mmWave bands.

In this study, the propagation measurements intended to characterize broadband mmWave wireless channels in two suburban macrocell environments are conducted for both LoS and OLoS links in the $28 \mathrm{GHz}$ and $39 \mathrm{GHz}$ bands. It is desirable for thousands of directional power delay profiles (PDPs) to be collected in different azimuth and elevation planes so that a comprehensive analysis of directional and omnidirectional mmWave propagation characteristics is performed. The largescale and small-scale fading statistics of mmWave radio propagation channels for suburban cellular systems in combination with foliage effects are analyzed and modeled. Frequencydependent and site-specific channel parameters are presented to facilitate cost-effective mmWave network deployment with enhanced coverage. A detailed analysis of the effects of foliage obstruction is presented considering the stochastic behavior of forward scattering clusters extracted from the cellular-type measurement data in vegetated suburban OLoS scenarios.

The remainder of this paper is organized as follows. In Section II, we present a detailed description of mmWave channel measurement campaigns in two suburban environments. The measurement results and analyses, with a focus on largescale and small-scale channel characteristics, such as path loss, shadow fading, composite and cluster-level channel dispersion, and foliage scattering, are reported in Sections III and IV, respectively. Finally, Section V concludes this work.

\section{Measurement CAMPAignS in Suburban MACROCELL SCENARIOS}

\section{A. Measurement Setup}

The propagation measurements are carried out with a commercial off-the-shelf (COTS) instrument-based flexible channel sounder [9]. At the TX side, 300 Mcps baseband Golay complementary sequences with a length of 4096 are generated and up-converted in an R\&S SMW200A vector signal generator, yielding a radio frequency (RF) null-to-null bandwidth of $600 \mathrm{MHz}$. Subsequently, the transmitted signals are amplified by two custom-made power amplifiers operating at $28 \mathrm{GHz}$ and $39 \mathrm{GHz}$, respectively. A wideband widebeam horn antenna with gain of $11.4 \mathrm{dBi}$ and half-power beamwidth (HPBW) of $60^{\circ}$ at $28 \mathrm{GHz}$ and gain of 18.7 $\mathrm{dBi}$ and $\mathrm{HPBW}$ of $50^{\circ}$ at $39 \mathrm{GHz}$ is employed for sector coverage, corresponding to TX total equivalent isotropically radiated power (EIRP) of $40.5 \mathrm{dBm}$ and $45.7 \mathrm{dBm}$ for the two concerned bands, respectively. At the $\mathrm{RX}$ side, the received signals are first amplified by low-noise amplifiers and then down-converted from RF to baseband via an R\&S FSW50 vector signal analyzer. Note that the RX is equipped with narrow beam horn antennas with gain of $25.6 \mathrm{dBi}$, HPBW of $9.5^{\circ}$ in azimuth and $9^{\circ}$ in elevation at $28 \mathrm{GHz}$, and with 27.7 $\mathrm{dB}$ gain, HPBW of $9.5^{\circ}$ in azimuth and $9.5^{\circ}$ in elevation at 39 $\mathrm{GHz}$, which can be mechanically steered with arbitrary angular resolution (using increments of $10^{\circ}$ here) in the azimuth and elevation planes.

Thanks to the GPS-disciplined Rubidium ( $\mathrm{Rb}$ ) frequency and timing references, the TX and RX can be separated for outdoor long-range measurements with precise clock synchronization and data acquisition. The back-to-back measurements via physically connecting the TX and RX RF ports are conducted before the on-site measurements for system calibration. The maximum measurable path losses for the directional channel (i.e., without beam combining) using such measurement setups are $162 \mathrm{~dB}$ and $168 \mathrm{~dB}$ at $28 \mathrm{GHz}$ and 39 $\mathrm{GHz}$, respectively. A channel sounder with these specifications can provide a multipath delay resolution of 3.3 ns. Further details on the COTS instrument-based channel sounder can be found in [9].

\section{B. Measurement Locations and Procedures}

As shown in Fig. 1, the measurements were performed in a villa district and an industrial park, both in Nanjing, China, in Fall 2018. For all the measurements, the TX antenna was installed on flat rooftops of low-rise buildings with fixed azimuth and elevation angles of departure (AOD/EOD) ${ }^{1}$, and the boresight of the TX sectors was directed toward the RX regions. At each RX location, the entire $2 \pi$ azimuth was swept for five fixed elevation angles of arrival (EOA) using directional antennas placed at a height of $1.9 \mathrm{~m}$. The angular steps were $10^{\circ}$ in both the azimuth $\phi$ and elevation $\theta$, corresponding to a total of $180(36 \times 5)$ directional PDPs recorded per TX-RX combination. Under such conditions, most direct and reflected signals arrive within this scanning angular range based on the geometry of the measurement locations. Note that the channels under measurement were assumed to be wide-sense stationary during such a scan duration (i.e., approximately $180 \mathrm{~s}$ ) because

\footnotetext{
${ }^{1}$ Following the standard jargon in [35], zenith angle and elevation angle have the same meaning, which denote the angle between the vertical plane and the direction of maximum antenna radiation measured in the vertical plane. In this paper, a unified description of the elevation angle is used and Fig. 1(c) shows the Cartesian coordinate system defined in this work.
} 


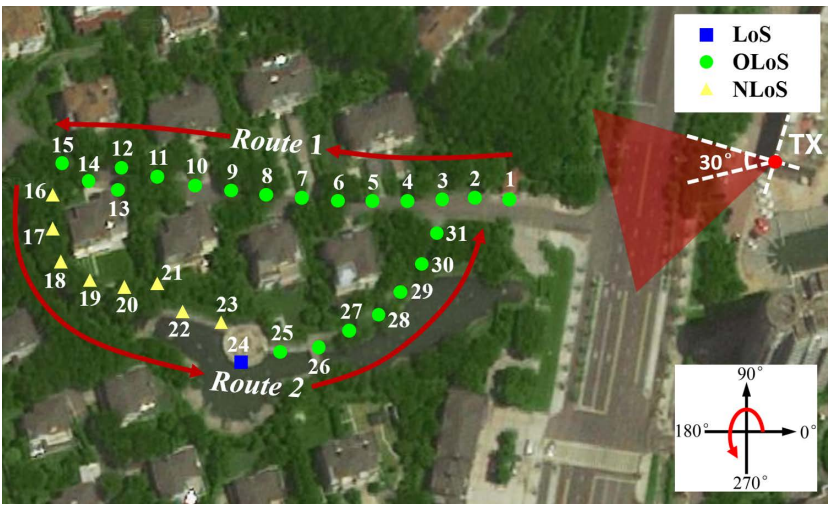

(a)

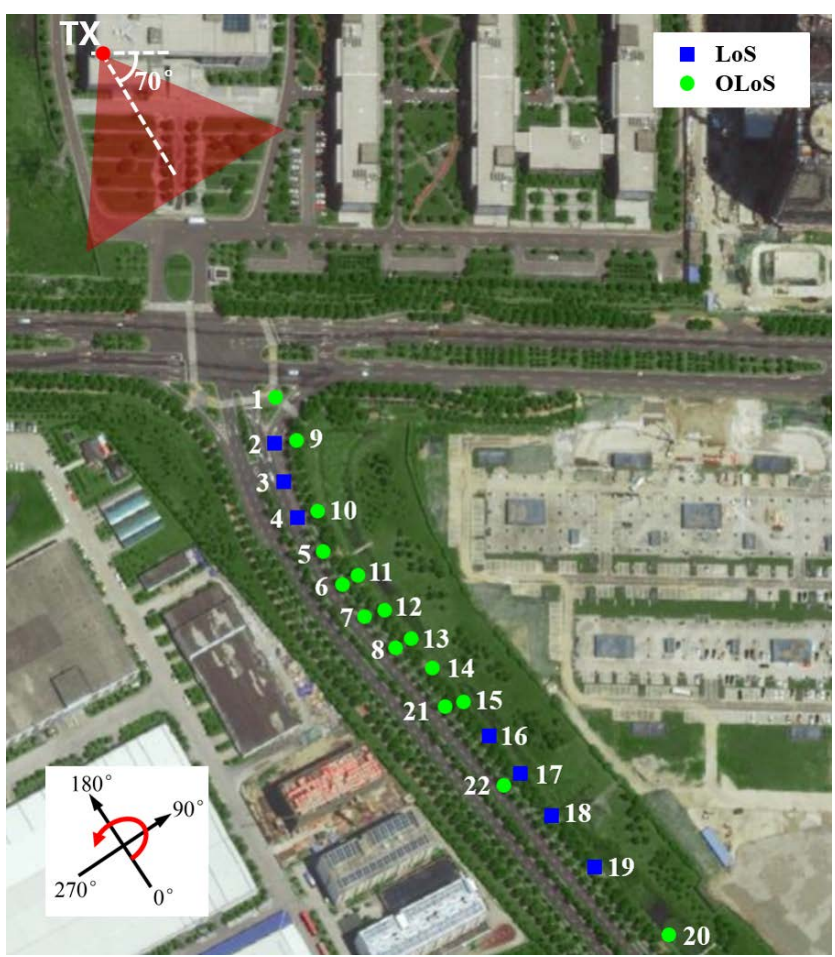

(b)

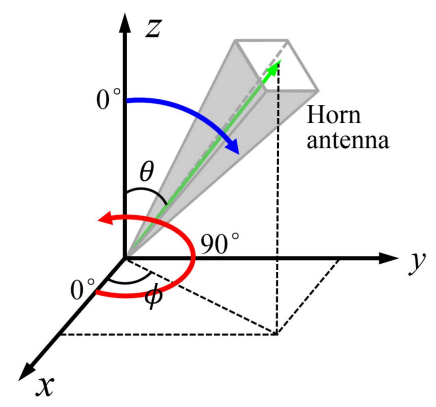

(c)

Fig. 1. The layout of the TX and RX locations in suburban environments, where the red dots represent the TX locations on the rooftops of buildings and the blue squares, green dots, and yellow triangles represent the LoS-, OLoS-, and NLoS-RX locations, respectively. (a) Villa district. (b) Industrial park. (c) The Cartesian coordinate system defined in this work.

the measurement campaigns were conducted in suburban areas during working hours, indicating minimal moving scatterers (e.g., vehicles and pedestrians) around the transceivers.

As mentioned above, the comparison of propagation charac- teristics in the two types of suburban scenarios with different types and fractions of vegetation cover is presented. Thus, two measurement locations are considered as follows.

1) Villa District: Fig. 1(a) depicts a typical suburban villa district populated with three-story houses, evergreen arbors, and shrubs. The average tree height in this area is approximately $6 \mathrm{~m}$ and the average height of the three-story houses with pitched roofs is about $10.5 \mathrm{~m}$. The TX antenna is deployed at a height of $15.4 \mathrm{~m}$ with a downtilt of $-5^{\circ}$, similar to a real suburban macrocell BS, whereby the RX antennas are automatically set to five EOAs (i.e., $70^{\circ}, 80^{\circ}, 90^{\circ}, 100^{\circ}$, and $110^{\circ}$ ). A total of $31 \mathrm{RX}$ positions are selected in densely vegetated areas, where RX 1-15 are along a street containing evergreen trees on the roadside (Route 1), RX 16-31 are along a path covered by low shrubs (Route 2), and RX 24 is a LoS reference location with a propagation distance of $171 \mathrm{~m}$. Note that RX 16-23 are blocked by the three-story villas. The range of the TX-RX separation distance varies from $83 \mathrm{~m}$ to $225 \mathrm{~m}$.

2) Industrial Park: This scenario is a typical suburban area constituting open spaces and the strong presence of deciduous broad-leaved trees with an average height of $10 \mathrm{~m}$. As shown in Fig. 1(b), the TX is placed at a height of $26.4 \mathrm{~m}$ with a antenna downtilt of $-15^{\circ}$, and $22 \mathrm{RX}$ locations along the Zijin Road are selected, including 7 LoS locations with a TXRX separation ranging from $220 \mathrm{~m}$ to $460 \mathrm{~m}$ and $15 \mathrm{OLoS}$ locations ranging from $200 \mathrm{~m}$ to $530 \mathrm{~m}$. At each measurement point, five EOAs (i.e., $80^{\circ}, 90^{\circ}, 100^{\circ}, 110^{\circ}$, and $120^{\circ}$ ) are considered based on the topology of the transceiver positions. Table I summarises the specifications for all RX positions and quantitative information on the vegetation. The distinguishing features of the industrial park in comparison with the villa district include 1) lower vegetation density and 2) no building blockages (i.e., presenting only LoS and OLoS situations).

\section{LARGe-Scale Channel Characterization}

\section{A. Basic Distance-Dependent Path Loss Model}

Path loss models are crucial for predicting the coverage probability of wireless communication systems with regard to received signal strength and interference. Fitting the proposed path loss models requires sufficient empirical path loss data to be obtained prior and is computed based on the received power, antenna and system gains, and spreading gain.

1) Data Pre-Processing: Following the recommendation in [36], the $i$ th strongest received power $P_{i}$ of the directional channel impulse response (CIR) $h_{i}(\tau)(i=1,2, \ldots, N)$ is collected over multiple delays:

$$
P_{i}[\mathrm{~mW}]=\int_{\tau_{0}}^{\tau_{1}}\left|h_{i}(\tau)\right|^{2} \mathrm{~d} \tau
$$

where $N$ denotes the number of the strongest beam of interest, and $\tau_{0}$ and $\tau_{1}$ denote the start and end of the PDP above the noise floor, which can be estimated using our method proposed in [7]. Here, the threshold value of $10 \mathrm{~dB}$ above the noise floor is utilized. By employing the obtained received power, the corresponding directional path loss can be calculated as follows:

$$
L_{i}[\mathrm{~dB}]=P_{\text {out }}+G_{\mathrm{T}}+G_{\mathrm{R}}+G_{\mathrm{S}}-10 \log _{10} P_{i}
$$


TABLE I

Specifications of Measurement Environments, RX Positions, and TX-RX SeParation Distances

\begin{tabular}{|c|c|c|c|c|c|c|c|}
\hline \multirow{2}{*}{ Environment } & \multirow{2}{*}{ Scenario } & \multicolumn{4}{|c|}{ Obstacle } & \multirow{2}{*}{ RX Position } & \multirow{2}{*}{ Range $[\mathrm{m}]$} \\
\hline & & Name & Height $[\mathrm{m}]$ & Crown diameter $[\mathrm{m}]$ & Intervals $[\mathrm{m}]$ & & \\
\hline \multirow{4}{*}{$\begin{array}{c}\text { Villa } \\
\text { district }\end{array}$} & LoS & I & l & I & I & 24 & 171 \\
\hline & \multirow{2}{*}{ OLoS } & Camphor trees & 6 & 6.5 & 7.5 & 1-15 (Route 1) & $83-208$ \\
\hline & & Buxus microphylla & 3.5 & 2.5 & 2 & 25-31 (Route 2) & $107-165$ \\
\hline & NLoS & Houses & 10.5 & 1 & 20 & 16-23 (Route 2) & $179-225$ \\
\hline \multirow{2}{*}{$\begin{array}{l}\text { Industrial } \\
\text { park }\end{array}$} & LoS & I & I & I & I & $2-4,16-19$ & $220-461$ \\
\hline & OLoS & Phoenix trees & 10 & 3.5 & 5 & $1,5-15,20-22$ & $201-530$ \\
\hline
\end{tabular}

where $P_{\text {out }}$ is the output power of the vector signal generator, $G_{\mathrm{T}}$ and $G_{\mathrm{R}}$ are the deembedded TX and RX antenna gains, and $G_{\mathrm{S}}$ is the system gain obtained via back-to-back calibration measurement. Note that the omnidirectional received power is not originally recorded but rather is non-coherently synthesised by the directional received power over all angular directions [5]

$$
P_{\text {omni }}[\mathrm{mW}]=\sum_{i=1}^{N} P_{i}
$$

Here, $N$ is $180(36 \times 5)$ for the omnidirectional case, and $P_{\text {omni }}$ is used to substitute for $P_{i}$ in (2) in computing the omnidirectional path loss. In this work, there are a total of 31 TX-RX location combinations for villa district, of which 28 and 27 locations have a $10 \mathrm{~dB}$ signal-to-noise ratio (SNR) for statistical channel modeling at $28 \mathrm{GHz}$ and $39 \mathrm{GHz}$, respectively; likewise, 21 and 16 effective TX-RX location combinations out of a total of 22 data sets are utilized for industrial park in these two bands. Moreover, the impact of the TX wide-beam antenna pattern is deembedded based on field-measured patterns in anechoic chambers and recorded angular misalignment.

2) Baseline Suburban Path Loss Models: The widely used CI model not only provides the benefit of easily comparing path loss across different frequency bands and environments but also has a physical basis in eliminating the uncertainty of measurements in the near field [5]. The CI model at the free space reference distance $d_{0} \mathrm{~m}$ is given by

$$
L_{\mathrm{CI}}(d)[\mathrm{dB}]=L_{o}\left(d_{0}\right)+10 n_{\mathrm{CI}} \log _{10}\left(d / d_{0}\right)+X_{\mathrm{CI}}
$$

where $L_{o}\left(d_{0}\right)[\mathrm{dB}]=20 \log _{10}\left(4 \pi d_{0} / \lambda\right)$ is the free space path loss with a wavelength $\lambda$ in meters, $n_{\mathrm{CI}}$ is the PLE computed via minimum mean square error fitting, and $X_{\mathrm{CI}}$ is a zeromean Gaussian random variable with standard deviation $\sigma_{\mathrm{CI}}$ in $\mathrm{dB}$. The reference distance $d_{0}$ is generally much larger in the traditional microwave bands (e.g., $1 \mathrm{~km}$ for large urban mobile systems, $100 \mathrm{~m}$ for microcell systems, and $1 \mathrm{~m}$ for indoor systems). Considering the radio zone of mmWave systems will become microcell or picocell, simple setting of $d_{0}=1 \mathrm{~m}$ would make comparisons of measurements and models simpler and provide a standard definition for the PLE in mmWave bands [5].

As mentioned above, several NLoS locations blocked by houses (deep fading region) result in outages in the villa district because of the lack of detectable MPCs, corresponding to the maximum TX-RX separation distances of $221 \mathrm{~m}$ and $209 \mathrm{~m}$ at $28 \mathrm{GHz}$ and $39 \mathrm{GHz}$, respectively. However, in the industrial park environment, the cell radii for the OLoS scenario are significantly larger than those in the villa district (more than $200 \mathrm{~m}$ ). Figs. 2 and 3 show the fitting curves of the CI models derived from the real-world measurement data at 28 $\mathrm{GHz}$ and $39 \mathrm{GHz}$ in the villa district and industrial park using the same measurement specifications, respectively. Note that no more than 50 effective directional path loss points (i.e., red and green markers) are shown for each RX location. Table II presents the PLEs and shadowing factors for the single-beam case with maximum directional received power (-best) and full-dimension non-coherent beam combining case (-omni). The $L_{o}\left(d_{0}\right)$ values are $61.38 \mathrm{~dB}$ and $64.26 \mathrm{~dB}$ at $28 \mathrm{GHz}$ and $39 \mathrm{GHz}$, respectively. Intuitively, the PLEs for the two suburban macrocell environments are all larger than the free space PLE of 2 and increase rapidly with increasing carrier frequency under all measurement settings.

In the Route 1 case of the villa district with the OLoS-omni link, $\left(n_{\mathrm{CI}}, \sigma_{\mathrm{CI}}\right)$ are $(3.28,3.37 \mathrm{~dB})$ and $(3.37,3.42 \mathrm{~dB})$ at these two mmWave bands. In the Route 2 case with OLoS-omni, the PLE $n_{\mathrm{CI}}$ is respectively 2.77 and 2.86, which is smaller than that in the Route 1 case, whereas the shadowing factor $\sigma_{\mathrm{CI}}$ is $4.26 \mathrm{~dB}$ and $3.34 \mathrm{~dB}$. This is probably because 1) Route 1 is blocked by dense camphor trees and experiences longer foliage depths, leading to severe vegetation attenuation compared with Route 2, which is mainly blocked by low shrubs and distant trees, and 2) the positions of RX $1-15$ along a straight line reduces the impact of the receiver siting on large-scale fading; however, the channel state information for mmWave cellular systems will change as the RX moves along a curved path (e.g., RX 25-31 in Route 2) considering their sensitivity to the environment. In the NLoS scenario, the omni-PLE is 3.43 at 28 $\mathrm{GHz}$, which is substantially higher for two OLoS conditions due to house blockage, whereas the NLoS-PLE of 3.45 is slightly higher than the PLEs in the OLoS settings at $39 \mathrm{GHz}$. To the best of our knowledge, diffracted signals over building rooftops are weak when the TX-RX separation is relatively long for NLoS links. This observation indicates that mmWave cellular systems do not work well in real-life dense-vegetated suburban deployment scenarios when the BS antenna heights are relatively low and when mobile users are located in deep fading regions.

In the industrial park environment, the PLE $n_{\mathrm{CI}}$ for the omni-LoS link at $39 \mathrm{GHz}$ is 2.15 , which is larger than the result of 2.01 at $28 \mathrm{GHz}$, and the path loss is about $4 \mathrm{~dB}$ higher than the theoretical free-space path loss. Similar behavior can also be observed from LoS reference measurements in the villa 


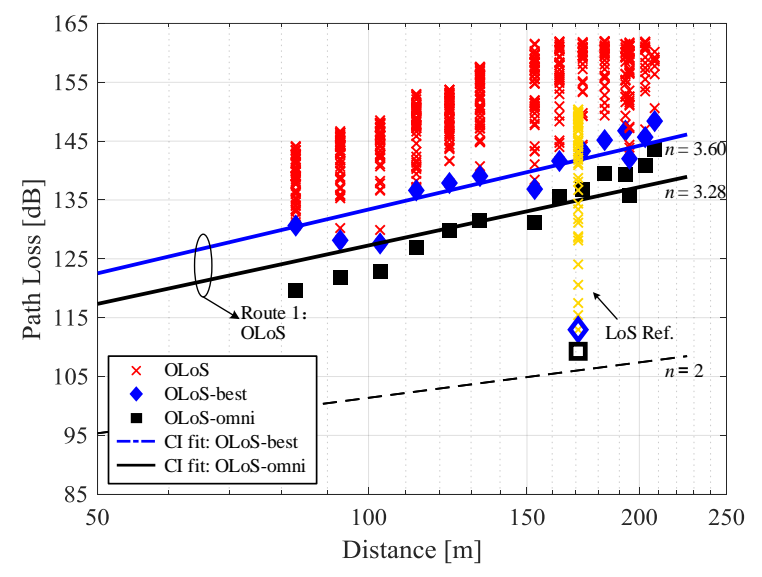

(a)

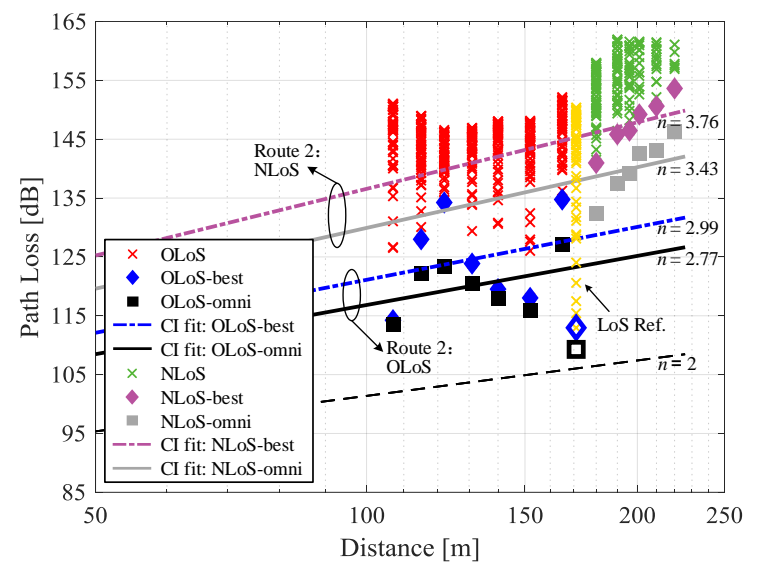

(c)

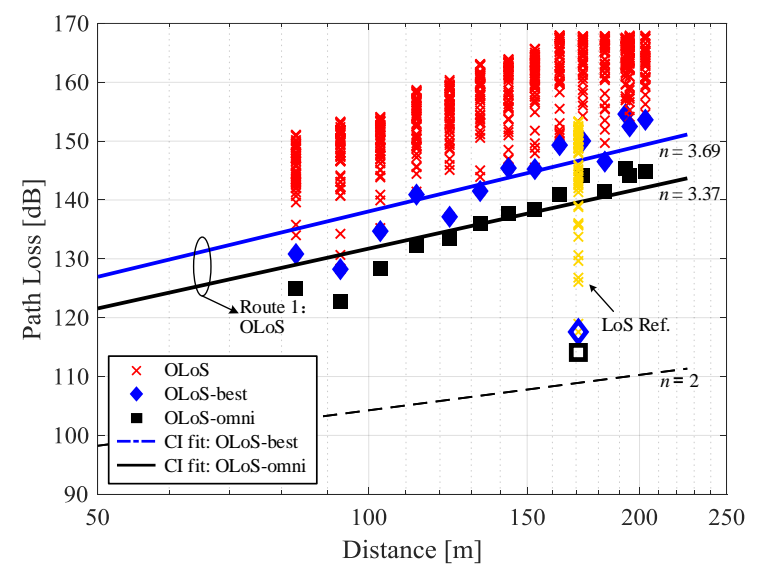

(b)

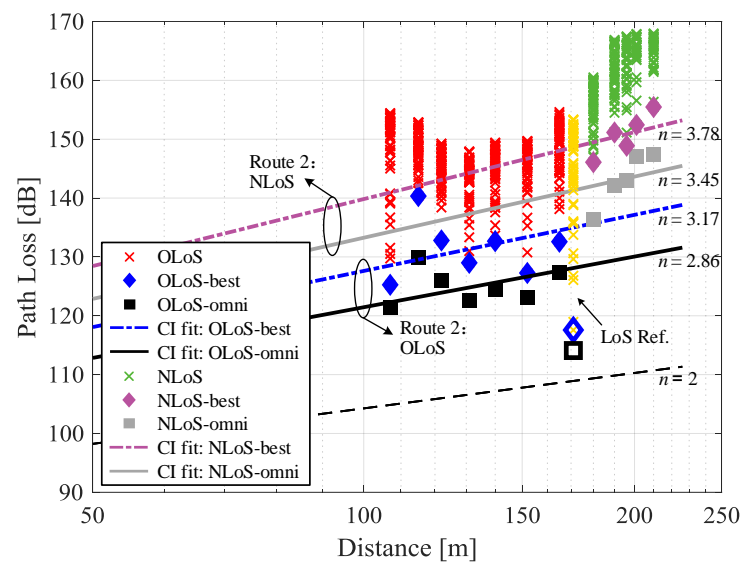

(d)

Fig. 2. The basic distance-dependent CI models in the villa district environment for the Route 1 OLoS link at (a) $28 \mathrm{GHz}$ and (b) $39 \mathrm{GHz}$ and the Route 2 OLoS and NLoS links at (c) $28 \mathrm{GHz}$ and (d) $39 \mathrm{GHz}$. Note that the yellow markers, empty diamond and empty square represent LoS-directional, LoS-best, and LoS-omni path losses, respectively.

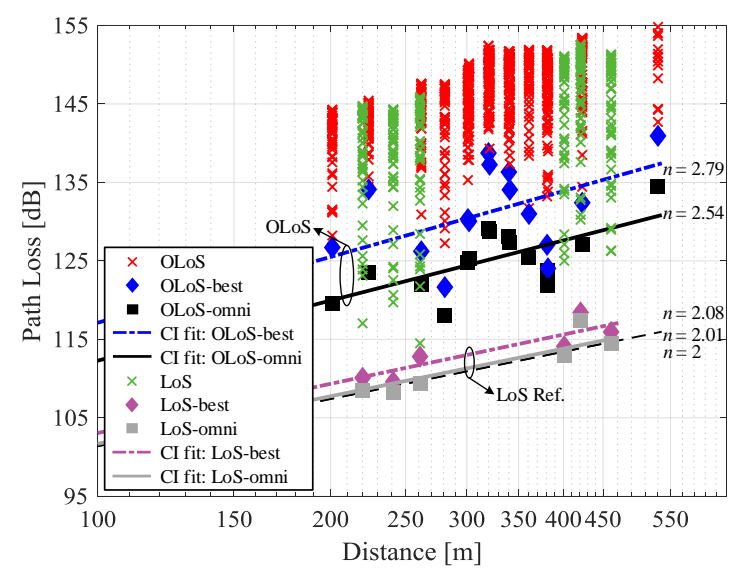

(a)

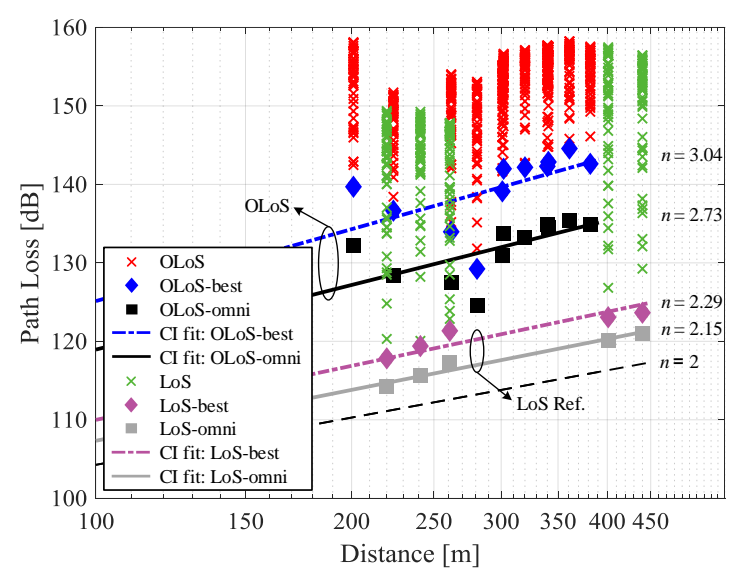

(b)

Fig. 3. The basic distance-dependent CI models for LoS and OLoS links in the industrial park environment at (a) $28 \mathrm{GHz}$ and (b) $39 \mathrm{GHz}$.

district and the results at $39 \mathrm{GHz}$ in [37]. In comparison with the measurement results in the villa district, the path loss for OLoS links in the industrial park environment is considerably low for three main reasons: 1) Propagation through denseleaved trees (e.g., camphor trees) likely results in larger foliage attenuation compared with sparse broad-leaved trees (e.g., phoenix trees), which is also in agreement with the empirical analysis under diversified vegetation [15], [27]. 2) The higher canopy cover is restricted to the villa district (see Fig. 1), corresponding to longer foliage depths with the same TXRX separation. Finally, 3) the path loss may decrease with increasing TX antenna height (within a reasonable range) 
TABLE II

Path Loss Model Parameters for Two Suburban Environments in 28 GHz and 39 GHz Bands

\begin{tabular}{|c|c|c|c|c|c|c|c|c|c|c|c|c|c|}
\hline \multirow{2}{*}{\multicolumn{2}{|c|}{$\begin{array}{c}\text { Environment } \\
\text { Scenario }\end{array}$}} & \multicolumn{8}{|c|}{ Villa district } & \multicolumn{4}{|c|}{ Industrial park } \\
\hline & & \multicolumn{2}{|c|}{ Route $1 \mathrm{OLoS}$} & \multicolumn{2}{|c|}{ Route 2 OLoS } & \multicolumn{2}{|c|}{ Route 2 NLoS } & \multicolumn{2}{|c|}{ All OLoS } & \multicolumn{2}{|c|}{ LoS } & \multicolumn{2}{|c|}{ OLoS } \\
\hline \multicolumn{2}{|c|}{ Frequency [GHz] } & 28 & 39 & 28 & 39 & 28 & 39 & 28 & 39 & 28 & 39 & 28 & 39 \\
\hline \multicolumn{14}{|c|}{ Basic distance-dependent CI model for $d_{0}=1 \mathrm{~m}$} \\
\hline \multicolumn{2}{|c|}{ Valid range $[\mathrm{m}]$} & $\leq 208$ & $\leq 203$ & $>165$ & $>165$ & $\leq 220$ & $\leq 210$ & $\leq 208$ & $\leq 203$ & $>461$ & $>440$ & $\leq 530$ & $\leq 382$ \\
\hline \multirow{2}{*}{ Dir. best } & $n_{\mathrm{CI}}$ & 3.60 & 3.69 & 2.99 & 3.17 & 3.76 & 3.78 & 3.40 & 3.52 & 2.08 & 2.29 & 2.79 & 3.04 \\
\hline & $\sigma_{\mathrm{CI}}[\mathrm{dB}]$ & 2.85 & 4.13 & 7.71 & 5.48 & 3.24 & 2.71 & 8.01 & 7.09 & 1.54 & 1.15 & 5.26 & 3.91 \\
\hline \multirow{2}{*}{ Omni. } & $n_{\mathrm{CI}}$ & 3.28 & 3.37 & 2.77 & 2.86 & 3.43 & 3.45 & 3.12 & 3.21 & 2.01 & 2.15 & 2.54 & 2.73 \\
\hline & $\sigma_{\mathrm{CI}}[\mathrm{dB}]$ & 3.37 & 3.15 & 4.26 & 3.34 & 3.89 & 3.63 & 6.54 & 6.28 & 1.58 & 0.58 & 3.04 & 2.93 \\
\hline
\end{tabular}

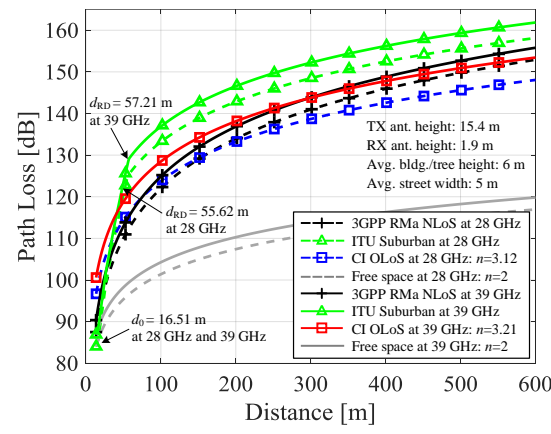

(a)

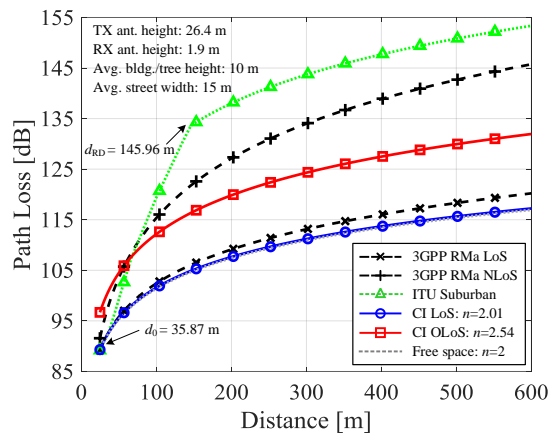

(b)

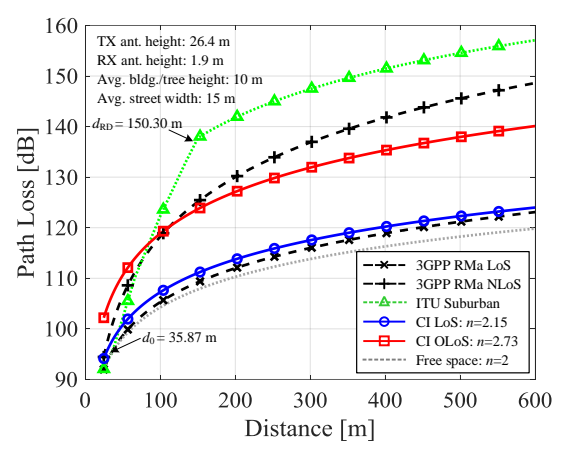

(c)

Fig. 4. Comparison of the proposed measurement-based CI models with ITU-R suburban site-specific path loss model [18] and 3GPP RMa path loss models [35]. (a) The OLoS link in the villa district environment. The LoS and OLoS links in the industrial park environment at (b) $28 \mathrm{GHz}$ and (c) $39 \mathrm{GHz}$.

when the direct path is blocked, as also documented in [24].

In Figs. 2 and 3, the PLEs for omnidirectional channels are substantially lower than the PLEs for the case of using optimal single beam with strongest received power, beam combining is thus needed to realize sufficient spatial diversity gains. Moreover, there are large differences in the directional path loss and the variations over different orientations of RX horn antenna are very noticeable, especially for LoS link. It is the fact that rotated horn antenna in the azimuth and elevation planes constructs a virtual spherical array, meaning that received signals from different antenna elements experience different propagation path and channel gains.

\section{B. Comparison of Standard Path Loss Models}

In Recommendation ITU-R P.1411 [18], a site-specific path loss model is proposed for propagation over rooftops in suburban NLoS areas, where it can be divided into three parts considering the dominant propagation mechanism in different regions. For example, direct waves can arrive at the RX when there is no obstacle for $d<d_{0}{ }^{2}$, several-time reflected waves are dominant when the TX-RX separation is relatively short for $d_{0} \leq d<d_{\mathrm{RD}}$, and diffracted waves are dominant when TX-RX separation is long for $d \geq d_{\mathrm{RD}}$. In addition, although 3GPP TR 38.901 does not provide a path loss model for

\footnotetext{
${ }^{2}$ Note that the $d_{0}$ in the ITU-R suburban site-specific path loss model has totally different meaning in comparison with the $d_{0}$ in the CI model. The $d_{0}$ in the ITU-R suburban model denotes the boundary between direct wave dominant region and reflected wave dominant region [18]. This suburban model was originally proposed in [38], which tried to develop a CI model in terms of appropriate setting of $d_{0}$ under various conditions.
}

suburban environments, it has promoted a model for rural macrocell (RMa) deployment scenarios up to $7 \mathrm{GHz}$ [35]. Both models are applicable for omnidirectional channels and need to specify several environmental parameters such as the TX and RX antenna heights, average building height, average street width, and street orientation with respect to the direct path. To compare with the measurement-based CI models proposed in Section III-A, the same configurations are used for the ITU suburban and 3GPP RMa models.

As shown in Fig. 4(a), the proposed CI models are compared with standard models in the villa district environment, where the PLEs are estimated over all OLoS TX-RX combinations. Intuitively, the CI models with PLEs of 3.12 and 3.21 are in general consistent with the 3GPP RMa NLoS models for the $28 \mathrm{GHz}$ and $39 \mathrm{GHz}$ channels, respectively; however, ITU$\mathrm{R}$ models will overestimate the path loss compared with the empirical-based CI models, especially when $d \geq d_{\mathrm{RD}}$. This observation indicates that the 3GPP RMa model for NLoS links is applicable up to tens of $\mathrm{GHz}$ (not merely up to 7 $\mathrm{GHz}$ as documented in [35]). Moreover, a substantially greater difference between the $28 \mathrm{GHz}$ and $39 \mathrm{GHz}$ channels in terms of the CI model can be observed in comparison with the standard models. Figs. 4(b) and (c) show the modeling results in the industrial park environment at $28 \mathrm{GHz}$ and $39 \mathrm{GHz}$, respectively. The ITU-R suburban model and 3GPP RMa model both overestimate the path loss for the region of $d \geq d_{\mathrm{RD}}$ in the OLoS setting under the assumption that the effect of foliage blockage is similar to buildings regarding NLoS RXs, while a direct wave can actually propagate through the sparse foliage. In the LoS scenario, the 3GPP RMa LoS 
model is similar to the free space model $(n=2)$, which is consistent with the results from our on-site measurements in suburban environments.

Therefore, the main conclusions derived from our studies regarding to the basic distance-dependent $\mathrm{CI}$ path loss models are as follows. 1) There has been a general increase in PLEs with carrier frequency in all suburban measurement settings. 2) The vegetated suburban environments are not rich scattering environments compared with dense urban environments, which can be ascribed to a lack of strong reflections, in turn having larger PLEs and slight path loss differences between the optimal direction and the beam synthesizing cases [5], [6]. 3) The existing standard path loss models (e.g., the ITU-R suburban site-specific model and the 3GPP RMa model) can be used for link budget in densely vegetated areas (e.g., villa district); in other words, the effect of dense-leaved trees on propagation loss prediction is similar to that of buildings or houses with the same height. 4) The coverage of mmWave suburban cellular networks in OLoS scenarios is strongly dependent on environmental parameters such as vegetation cover, physical blockages, and BS antenna heights.

\section{Azimuth-Angle-Based Path Loss Characterization}

The basic distance-dependent single-slope path loss models (e.g., the CI model and the ABG model) are typically used to fit the measured path loss data and generally perform well when all RX locations are within the TX lit regions and when operating under the environments with consistent propagation characteristics. However, the shadow fading, described as a log-normal distributed random variable on the top of the classical power law path loss model, does not capture the effect of obstacles on mmWave radio propagation and in turn will derive misleading trends of various performance metrics [39]. For example, the shadowing factor $\sigma_{\mathrm{CI}}$ for Route $1 \mathrm{OLoS}$ and Route 2 NLoS links, on average, are lower than that for Route 2 OLoS links due to their susceptibility to the effects of physical objects in the mmWave band. On the other hand, the beamspace representation of the propagation channel is a natural choice for optimizing directional transmission schemes in mmWave communication systems under the employment of large-scale antenna arrays [2]. Overall, the $\sigma_{\mathrm{CI}}$ for the best directional channels are greater than the results for the omnidirectional channel, as identified in Table II. As shown in Figs. 2 and 3, the minimum directional path loss may even decrease with increasing distance for certain special cases, such as the Route 2 OLoS case in the villa district environment and the OLoS case with the TX-RX separation range of $[201,283] \mathrm{m}$ in the industrial park environment, as well as the similar results reported in [5], [21]. This observation implies a strong influence of propagation areas that are sparsely populated with a finite number of scatterers in the characterization of mmWave large-scale fading channels.

Therefore, to ensure reliable system-level performance analysis and coverage optimization of mmWave cellular networks under different transmission schemes, a sophisticated largescale fading characterization is expected assuming an azimuthangle-dependent $\mathrm{CI}$ (ACI) directional power law path loss, considering the impact of transceiver siting:

$$
\begin{aligned}
L_{\mathrm{ACI}}\left(d, \phi^{\mathrm{TX}}\right)[\mathrm{dB}]= & L_{o}\left(d_{0}\right)+10 n_{\mathrm{ACI}} \log _{10}\left(d / d_{0}\right) \\
& +g\left(\phi^{\mathrm{TX}}\right)+\eta\left(d, \phi^{\mathrm{TX}}\right)+X_{\mathrm{ACI}}
\end{aligned}
$$

where $\phi^{\mathrm{TX}}$ is the AOD, $L_{o}\left(d_{0}\right)$ is the theoretical free-space path loss given in (4), $n_{\mathrm{ACI}}$ is the PLE, and $X_{\mathrm{ACI}}$ is a zeromean Gaussian random variable with shadowing factor $\sigma_{\mathrm{ACI}}$ in $\mathrm{dB}$. The impact of the directional antenna patterns $g(\phi)$ in $\mathrm{dB}$ can be written as [40]

$$
g\left(\phi^{\mathrm{TX}}\right)[\mathrm{dB}]= \begin{cases}\frac{12\left(\phi^{\mathrm{TX}}-\phi_{o}\right)^{2}}{\omega^{2}}, & \text { for }\left|\phi^{\mathrm{TX}}-\phi_{o}\right| \leq \varphi \\ G-G_{\min }, & \text { for }\left|\phi^{\mathrm{TX}}-\phi_{o}\right|>\varphi\end{cases}
$$

where $\phi_{o}$ is the boresight direction of the TX antenna, $G$ and $\omega$ are the gain and HPBW of the TX antenna, respectively, $G_{\min }$ is the minimum antenna gain, and $\varphi=\omega \sqrt{G_{\min } / 12}$ is the angle that corresponds to the main lobe. On the other hand, $g\left(\phi^{\mathrm{TX}}\right)$ can alternatively consider the field-measured antenna patterns. The excess loss $\eta\left(d, \phi^{\mathrm{TX}}\right)$ is expressed as

$$
\eta\left(d, \phi^{\mathrm{TX}}\right)=L_{\mathrm{block}}(d) \cdot \mathbb{1}\left(\phi^{\mathrm{TX}}=\phi_{i}^{\mathrm{TX}}\right)
$$

where the probability function of existing blockages along the orientation of $\phi_{i}^{\mathrm{TX}}$ is $\mathbb{1}\left(\phi^{\mathrm{TX}}=\phi_{i}^{\mathrm{TX}}\right), \mathbb{1}(\cdot)$ denotes the indicator function, and $L_{\text {block }}(d)$ denotes the excess loss due to the presence of physical blockages. When (5) is actually used for system-level performance analysis and cell planning of mmWave cellular networks, $\phi_{i}^{\mathrm{TX}}$ is set as a deterministic value depending on blockage location. Note that blockage locations can be obtained based on the stochastic blockage models [39] or precise geographical information. In the OLoS setting, the $n_{\mathrm{ACI}}$ is equal to the $n_{\mathrm{CI}}$ extracted based on LoS reference measurements and the excess loss due to propagation through vegetation is modeled as [32]

$$
L_{\text {block }}(d)=A_{\mathrm{m}}\left[1-\exp \left(-d_{\mathrm{v}} \gamma / A_{\mathrm{m}}\right)\right]
$$

where $d_{\mathrm{v}}$ is the foliage depth with $d_{\mathrm{v}}<d, \gamma$ is the specific attenuation in $\mathrm{dB} / \mathrm{m}$ for very short vegetative paths, and $A_{\mathrm{m}}$ is the maximum attenuation for a specific type and depth of vegetation.

Intuitively, the advantage of the proposed analytical method in (5) is that it adopts a more practical consideration of blockage effects and mmWave beamspace channel characteristics. As illustrated in Fig. 5, there are three typical cases used for mmWave directional channel representation with a focus on the azimuth-angle-based large-scale fading characteristics. At the TX or BS side, a high-gain wide-beam directional antenna with an HPBW of $\omega$ or a phased array with limited steered range is utilized, corresponding to a fan-shaped coverage range. At the RX or user side, a beam scanning process using narrow beam patterns is considered to determine the directions of the effective signals. As for case $1, \operatorname{LoS}$ propagation is dominant and likely has a larger Ricean $K$-factor. We can also notice in Table II a quite small $\sigma_{\mathrm{CI}}$ for both the $28 \mathrm{GHz}$ and 39 $\mathrm{GHz}$ channels in the LoS reference measurements, as well as a slight difference between directional and omnidirectional path losses from Fig. 3. This result also proves the aforementioned assumption that shadowing will increase with the presence 


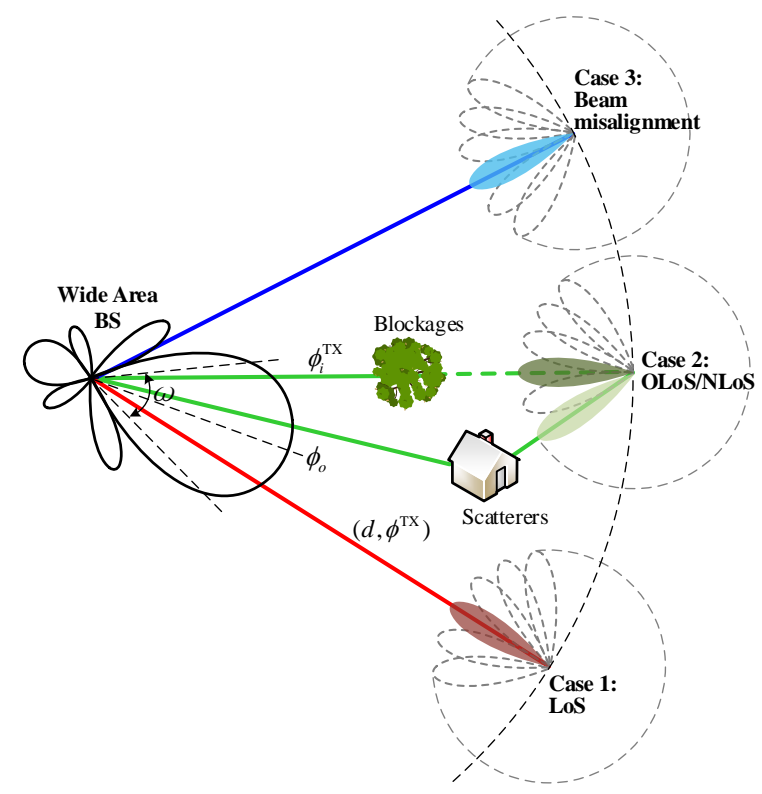

Fig. 5. Illustration of the three typical cases in mmWave directional transmission with a focus on large-scale path loss characterization.

of LoS obstacles. In the OLoS/NLoS case, the direct path is blocked by vegetation and buildings, whereby the building entry loss is remarkably larger than the foliage attenuation. However, the rich reflection and diffraction from the surrounding physical scatterers can aid in establishing stable communication links over the most promising directions. Cases 1 and 2 assume that the RX locations are within the lit region along the orientation of the TX antenna main lobe; however, in case 3 , the TX and RX beams are misaligned, which decreases the dynamic range of mmWave cellular systems by accounting for possible antenna gains. The ACI model can be further extended to a full-dimensional model considering the impact of depression angles and TX antenna heights on path loss [25], [41].

\section{SMALl-Scale ChanNel Characteristics}

\section{A. Cluster-Based Channel Characterization}

The clustering nature takes advantage of the beamspace representation of multipath channels, which plays a crucial role in the physical layer design of mmWave cellular wireless systems. To quantify mmWave beam domain propagation characteristics, the improved two-step KPowerMeans clustering algorithm is used to cluster effective MPCs [42]. The MPCs are defined as the peaks whose amplitudes are above the detection level $P_{\mathrm{D}}$ which is given by

$$
P_{\mathrm{D}}=\max \left\{P_{\max }-P_{\mathrm{th}}, N_{o}+\gamma\right\}
$$

where $P_{\max }$ is the maximum peak power over all directions, $P_{\text {th }}$ is the power threshold relative to the highest peak, and $\gamma$ is the SNR relative to the noise floor $N_{o}$. In this work, $P_{\text {th }}$ and $\gamma$ are respectively set as $25 \mathrm{~dB}$ and $20 \mathrm{~dB}$ to ensure each MPC within the detection level and above the noise floor. By employing the aforementioned dynamic detection threshold and peak detection algorithm, effective MPCs are estimated. Consequently, the $i$ th MPC within a cluster is
TABLE III

The Statistics of the SMall-Scale Channel Parameters for LoS AND OLOS LiNKS IN VEGETATED SUbURBAN MACROCELL ENVIRONMENTS

\begin{tabular}{|c|c|c|c|c|c|c|c|}
\hline \multirow{3}{*}{\multicolumn{2}{|c|}{$\begin{array}{c}\text { Environment } \\
\text { Scenario } \\
\text { Freq. [GHz] }\end{array}$}} & \multirow{2}{*}{\multicolumn{2}{|c|}{$\begin{array}{l}\text { Villa district } \\
\text { All OLoS }\end{array}$}} & \multicolumn{4}{|c|}{ Industrial park } \\
\hline & & & & \multicolumn{2}{|c|}{ LoS } & \multicolumn{2}{|c|}{ OLoS } \\
\hline & & 28 & 39 & 28 & 39 & 28 & 39 \\
\hline \multicolumn{8}{|c|}{ Composite channel parameters } \\
\hline \multirow{2}{*}{$\begin{array}{l}\sigma_{\tau} \\
{[\mathrm{ns}]}\end{array}$} & $\mu$ & 63.87 & 53.40 & 44.03 & 31.66 & 74.45 & 62.35 \\
\hline & $\sigma$ & 36.97 & 31.15 & 19.82 & 17.95 & 35.47 & 26.02 \\
\hline \multirow{2}{*}{$\begin{array}{c}\sigma_{\phi^{\mathrm{RX}}} \\
{\left[^{\circ}\right]} \\
\end{array}$} & $\mu$ & 33.63 & 29.97 & 15.99 & 13.80 & 44.32 & 40.94 \\
\hline & $\sigma$ & 13.23 & 12.54 & 7.03 & 8.53 & 10.38 & 10.11 \\
\hline \multirow{2}{*}{$\begin{array}{c}\sigma_{\theta^{\mathrm{RX}}} \\
{\left[^{\circ}\right]}\end{array}$} & $\mu$ & 9.17 & 8.70 & 7.93 & 7.68 & 10.31 & 8.86 \\
\hline & $\sigma$ & 1.94 & 1.47 & 1.77 & 2.29 & 2.48 & 2.06 \\
\hline \multicolumn{8}{|c|}{ Cluster-level channel parameters } \\
\hline \multirow{2}{*}{$M$} & $\mu$ & 4.46 & 3.35 & 3.50 & 2.60 & 4.80 & 3.82 \\
\hline & $\sigma$ & 1.56 & 0.86 & 1.38 & 0.55 & 1.66 & 0.60 \\
\hline \multirow{2}{*}{$\begin{array}{l}\sigma_{\mathrm{c}, \tau} \\
{[\mathrm{ns}]}\end{array}$} & $\mu$ & 21.01 & 17.44 & 16.50 & 16.28 & 24.18 & 18.92 \\
\hline & $\sigma$ & 11.93 & 9.30 & 9.54 & 6.37 & 12.06 & 10.58 \\
\hline \multirow{2}{*}{$\begin{array}{c}\sigma_{\mathrm{c}, \phi} \mathrm{RX} \\
{\left[{ }^{\circ}\right]}\end{array}$} & $\mu$ & 14.31 & 14.06 & 10.37 & 9.9 & 19.46 & 15.20 \\
\hline & $\sigma$ & 7.33 & 6.49 & 5.63 & 3.11 & 8.99 & 6.33 \\
\hline \multirow{2}{*}{$\begin{array}{c}\sigma_{\mathrm{c}, \theta} \mathrm{RX} \\
{\left[{ }^{\circ}\right]}\end{array}$} & $\mu$ & 7.33 & 7.31 & 7.04 & 6.85 & 7.88 & 7.48 \\
\hline & $\sigma$ & 1.81 & 1.92 & 2.71 & 1.10 & 2.02 & 2.11 \\
\hline
\end{tabular}

characterized by $\left(p_{i}, \tau_{i}, \phi_{i}^{\mathrm{RX}}, \theta_{i}^{\mathrm{RX}}\right)$, where $p_{i}$ is the path gain, $\tau_{i}$ is the absolute propagation delay, $\phi_{i}^{\mathrm{RX}}$ is the azimuth angle of arrival (AOA), and $\theta_{i}^{\mathrm{RX}}$ is the EOA. All these parameters are estimated via the algorithm developed in [43], resulting in a multipath delay resolution of $3.3 \mathrm{~ns}$ and an angular resolution of $10^{\circ}$, which are determined by the signal bandwidth and the angular resolution of the measured antenna patterns in use, respectively. Therefore, the RMS intra-cluster delay spread $\sigma_{\tau}$ is computed as

$$
\sigma_{\mathrm{c}, \tau}=\sqrt{\frac{\sum_{i} \tau_{i}^{2} p_{i}}{\sum_{i} p_{i}}-\left(\frac{\sum_{i} \tau_{i} p_{i}}{\sum_{i} p_{i}}\right)^{2}}
$$

The RMS intra-cluster azimuth angular spread of arrival (ASA) $\sigma_{\mathrm{c}, \phi^{\mathrm{Rx}}}$ is computed as [44]

$$
\sigma_{\mathrm{c}, \phi^{\mathrm{RX}}}=\sqrt{\frac{\sum_{i} \mid \exp \left(j \phi_{i}^{\mathrm{RX}}\right)-\mu_{\mathrm{c},\left.\phi_{\mathrm{Rx}}\right|^{2} p_{i}}}{\sum_{i} p_{i}}}
$$

and

$$
\mu_{\mathrm{c}, \phi^{\mathrm{RX}}}=\frac{\sum_{i} \exp \left(j \phi_{i}^{\mathrm{RX}}\right) p_{i}}{\sum_{i} p_{i}}
$$

By replacing $\phi^{\mathrm{RX}}$ with $\theta^{\mathrm{RX}}$, the elevation angular spread of arrival (ESA) $\sigma_{\mathrm{c}, \theta^{\mathrm{Rx}}}$ can be estimated. Furthermore, the composite channel dispersion parameters are also calculated following similar definitions in [44].

Table III identifies the composite and cluster-level channel parameters in the two suburban macrocell environments. In the industrial park environment, the composite delay and angular dispersion results for the OLoS link are significantly greater than the results for the LoS link and generally decrease with increasing carrier frequency. This result is comparable to the site-specific foliage measurement results at $24 \mathrm{GHz}$ and 28 $\mathrm{GHz}$ [28], [30]. As a comparison of the results in the villa district over all OLoS RX positions, propagation in sparse 


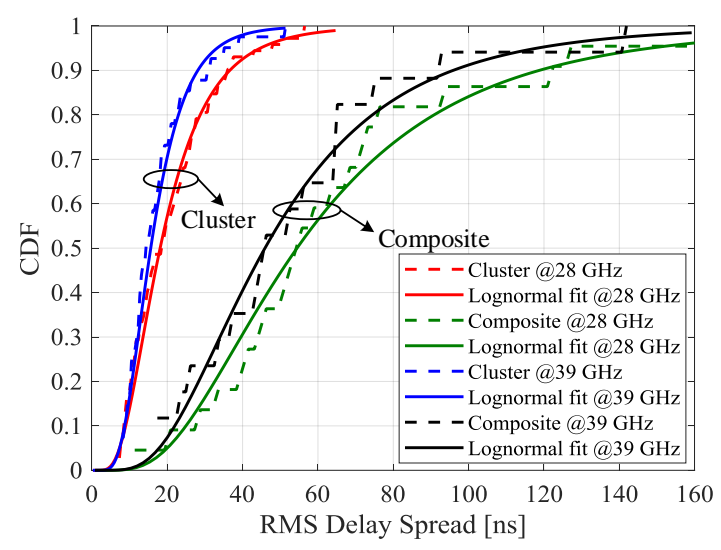

(a)

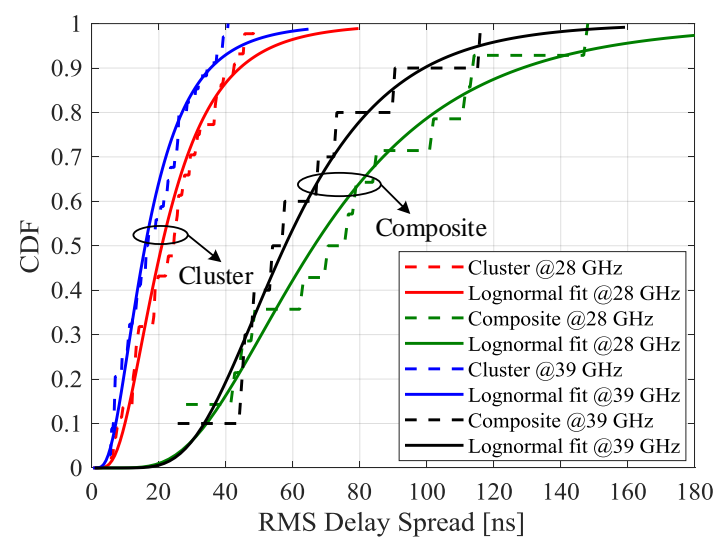

(b)

Fig. 6. The empirical and log-normal fitted CDF of the composite and cluster delay spread at $28 \mathrm{GHz}$ and $39 \mathrm{GHz}$ in the (a) villa district and (b) industrial park environments.

vegetated areas is likely to increase the delay and angular spreads due to the greater number of airy spaces, which will contribute to lower vegetation attenuation and increased forward MPCs. This observation is in-line with the assumption that vegetative areas may no longer be homogeneous random scattering media [45]. The means of the delay spread on the decibel scale (i.e., $\left.\log _{10}\left(\sigma_{\tau}[\mathrm{s}]\right)\right)$ are -7.39 and -7.18 at $28 \mathrm{GHz}$ for the industrial park LoS and OLoS scenarios, respectively. The corresponding mean values in the 3GPP models are 7.09 and -6.58 for urban macrocell (UMa) LoS and NLoS scenarios, which are remarkably larger than our results due to the rich reflections from surrounding buildings and are -7.49 and -7.43 for the RMa scenarios [35]. This trend can also be observed for RMS ASA and ZSA, indicating that vegetated suburban environments are not rich scattering environments. Consequently, fewer clusters $M$ can be extracted (see Table III) compared with urban environments [6], [35]. On the other hand, the standard deviations $\sigma$ of the composite delay and azimuth angular spreads in villa district environment are much larger because of the combination of two RX data sets measured along different routes.

Concerning the cluster-level dispersion parameters, the delay spreads at $28 \mathrm{GHz}$ in both suburban environments are significantly smaller than the results in the 3GPP UMa model of 42.63 ns for both LoS and NLoS links; conversely, they are

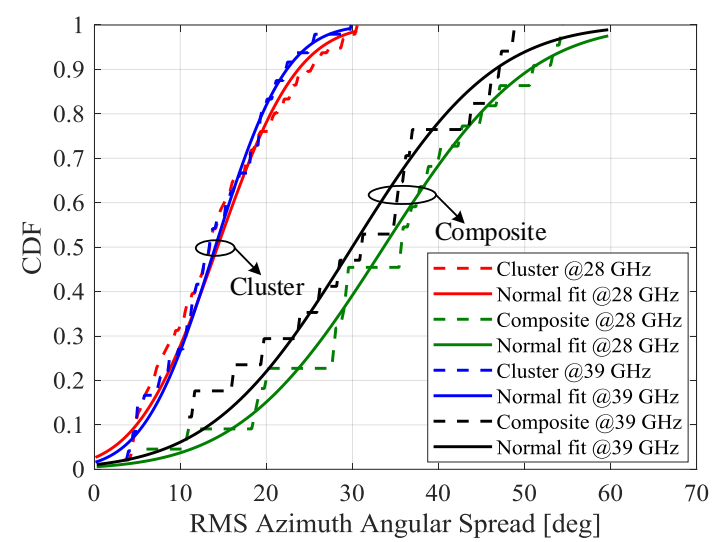

(a)

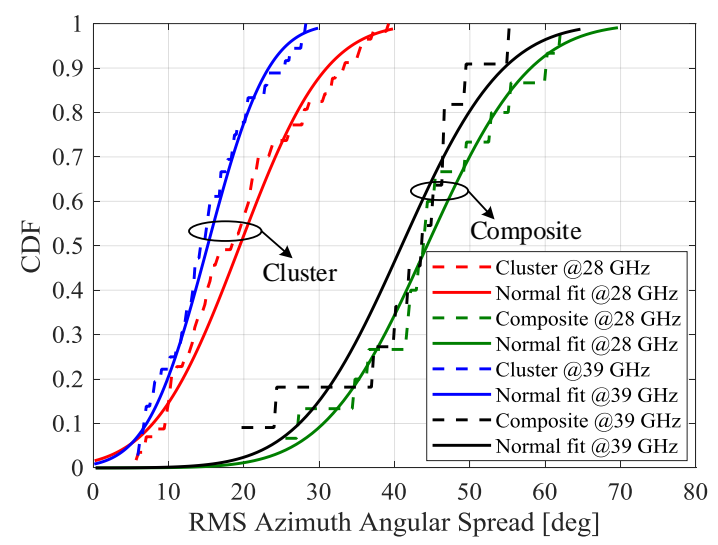

(b)

Fig. 7. The empirical and normal fitted CDF of composite and cluster ASA at $28 \mathrm{GHz}$ and $39 \mathrm{GHz}$ in the (a) villa district and (b) industrial park environments.

only $13.78 \mathrm{~ns}$ for the $39 \mathrm{GHz}$ channel, which is less than our results. The frequency-dependent means of the cluster delay spread, expressed as $\log _{10}\left(\sigma_{\mathrm{c}, \tau}\right)=a-b \log _{10} f,(a, b>0)$, hence need to be modified to have a smaller value of $b$. Fig. 6 shows the cumulative distribution functions (CDFs) of the composite and cluster delay spread along with the corresponding log-normal fits for different frequency bands and environments. The composite delay spreads differ considerably from the cluster-level results, while substantially greater differences can be found in the industrial park environment. This observation demonstrates that the temporal sparsity of mmWave measured channels is pronounced in suburban environments. Of the cluster ASA $\sigma_{\mathrm{c}, \phi^{\mathrm{Rx}}}$, our results in the OLoS setting are all greater than the default values in the 3GPP UMa and RMa NLoS channel models, whereas the means in the LoS setting are well aligned. We speculate that forward scattering through foliage probably increases the cluster angular spread, and its impact will be discussed later. Fig. 7 shows the empirical and normal fitted CDFs of ASA, where a slight difference in intra-cluster ASA can be observed between the 28 $\mathrm{GHz}$ and $39 \mathrm{GHz}$ channels in densely vegetated areas (e.g., the villa district), while approximately $4.25^{\circ}$ and $8.55^{\circ}$ differences exist for the cases with cumulative probabilities of $50 \%$ and $95 \%$ in sparsely vegetated areas (e.g., the industrial park). 


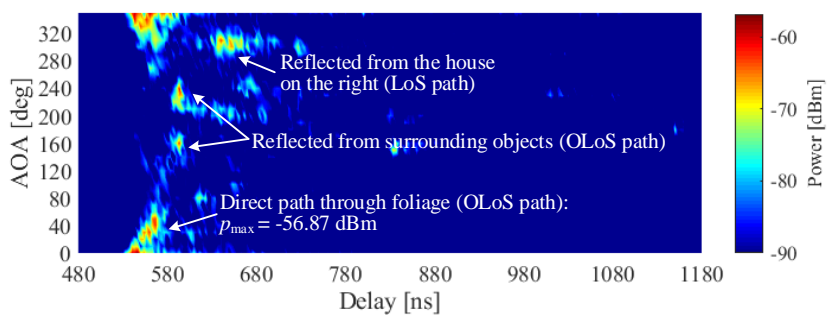

(a)

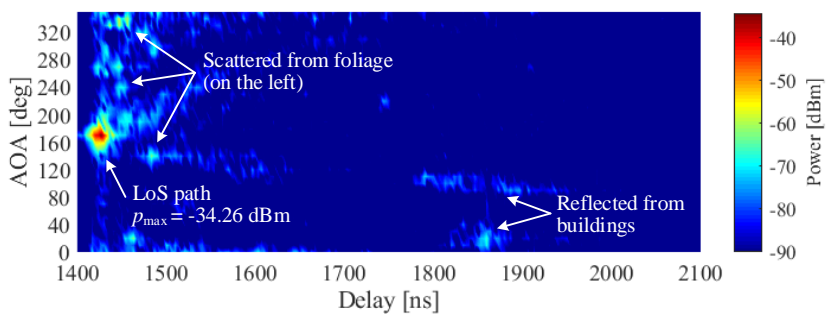

(b)

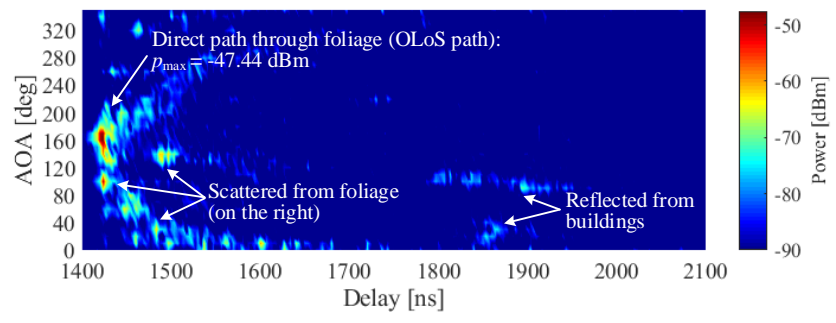

(c)

Fig. 8. Typical PDAPs at $28 \mathrm{GHz}$ for (a) RX 5 (OLoS, $d=123 \mathrm{~m}$ ) in the villa district environment and (b) RX 17 (LoS, $d=421 \mathrm{~m}$ ) and (c) RX 22 (OLoS, $d=423 \mathrm{~m}$ ) in the industrial park environment.

\section{B. Foliage Scattering and Its Impact}

Propagation through vegetation not only experiences large signal attenuation but also impacts the temporal and spatial dispersion of the OLoS path (i.e., the LoS path obstructed by the front foliage). Using the directional scanning sounding method, power delay angular profiles (PDAPs) can be applied to analyze the directional power distribution in the delay and angular domains. Fig. 8 shows several typical PDAPs in the two measured environments at $28 \mathrm{GHz}$ using the same measurement configurations. The mapping results of the clusters and the physical objects reveal that forward scattering by foliage is dominant for the OLoS link (see Figs. 8(a) and (c)) and likely increases the angular range and time duration of the major cluster compared with the LoS cluster.

To characterize the OLoS path relative to other stochastic paths regarding small-scale fading, the ratio of the power of the dominant cluster to that of the diffuse clusters is given by

$$
R[\mathrm{~dB}]=10 \log _{10} \frac{\sum_{i \in \mathcal{C}_{1}} p_{i}}{\sum_{m=2}^{M} \sum_{i \in \mathcal{C}_{m}} p_{i}}
$$

where the set $\mathcal{C}_{m}(m=1,2, \ldots, M)$ denotes the MPC indices belonging to the $m$ th cluster, $M$ denotes the number of clusters, and the set $\mathcal{C}_{1}$ denotes the dominant cluster, which has the strongest path power and lowest propagation delay. The power ratio as outlined by (13) can also be extended to the LoS setting, where the set $\mathcal{C}_{1}$ corresponds to the LoS
TABLE IV

The Statistics of the Cluster-Level Channel Parameters For THE FIRST-ARRIVAL OR DOMINANT CLUSTER

\begin{tabular}{c|c|c|c|c|c|c|c}
\hline \multicolumn{2}{c|}{ Environment } & \multicolumn{2}{c|}{ Villa district } & \multicolumn{4}{c}{ Industrial park } \\
\hline \multicolumn{2}{c}{ Scenario } & \multicolumn{2}{c|}{ All OLoS } & \multicolumn{2}{c}{ LoS } & \multicolumn{2}{c}{ OLoS } \\
\hline \multicolumn{2}{c|}{ Freq. [GHz] } & 28 & 39 & 28 & 39 & 28 & 39 \\
\hline \multirow{2}{*}{$\begin{array}{c}R \\
{[\mathrm{~dB}]}\end{array}$} & $\mu$ & 6.92 & 6.48 & 8.06 & 7.73 & 7.51 & 6.53 \\
\cline { 2 - 8 } & $\sigma$ & 1.51 & 1.44 & 2.77 & 2.24 & 1.34 & 1.20 \\
\hline$\sigma_{\tau}$ & $\mu$ & 13.83 & 11.25 & 5.49 & 4.94 & 13.31 & 10.95 \\
\cline { 2 - 9 }$[\mathrm{ns}]$ & $\sigma$ & 5.86 & 1.48 & 2.27 & 3.18 & 5.95 & 3.95 \\
\hline$\sigma_{\phi^{\mathrm{RX}}}$ & $\mu$ & 16.93 & 15.90 & 6.11 & 5.66 & 21.68 & 16.55 \\
\cline { 2 - 8 }$\left[{ }^{\circ}\right]$ & $\sigma$ & 5.90 & 6.20 & 2.55 & 2.18 & 5.62 & 5.73 \\
\hline \multirow{2}{*}{$\sigma_{\theta^{\mathrm{RX}}}$} & $\mu$ & 7.61 & 7.31 & 6.36 & 6.12 & 7.46 & 7.38 \\
\cline { 2 - 8 }$\left[{ }^{\circ}\right]$ & $\sigma$ & 1.98 & 1.42 & 1.45 & 1.46 & 1.14 & 2.04 \\
\hline \multirow{2}{*}{} & & & & & & &
\end{tabular}

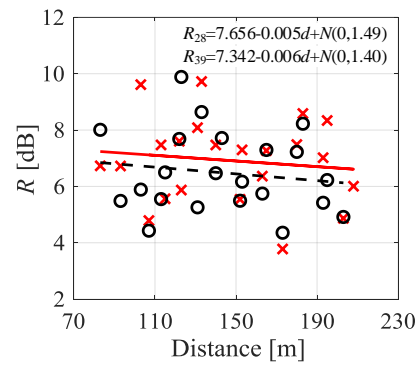

(a)

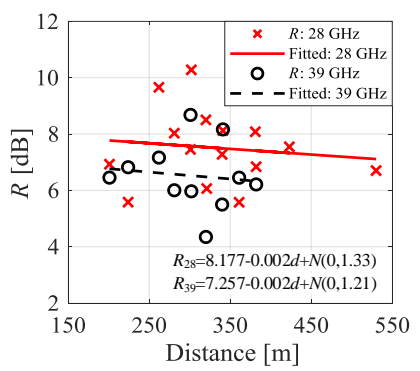

(b)
Fig. 9. Linear fitting of the power ratio $R$ with distance in the (a) villa district and (b) industrial park environments.

cluster. As reported in Table IV, the mean $R$ values are 8.06 $\mathrm{dB}$ and $7.73 \mathrm{~dB}$ for the occurrence of the LoS component at $28 \mathrm{GHz}$ and $39 \mathrm{GHz}$, respectively. These values are much higher than the mean values in the OLoS setting because the OLoS path experiences strong vegetation attenuation, while the powers of the reflected and scattered paths are relatively constant. This can be seen from Figs. 8(b) and (c), where RX 17 and 22 have approximately the same TX-RX separation distance; however, the maximum path powers are $-34.26 \mathrm{dBm}$ and $-47.44 \mathrm{dBm}$, respectively. Meanwhile, the not dramatically lower $R$ quantitatively verifies that the OLoS path is still dominant for OLoS links despite the vegetation attenuation being quite severe. To verify whether there is relationship between $R$ and propagation distance, Fig. 9 shows the linear fitting results in OLoS scenario with the model expressed as

$$
R(d)[\mathrm{dB}]=\alpha+\beta d+X_{R}
$$

where $\alpha$ is the intercept, $\beta$ is the slope, and $X_{R}$ is a Gaussian random variable $\sim \mathcal{N}\left(0, \sigma_{R}^{2}\right)$. The corresponding fitting parameters are also shown in Fig. 9. As the distance increases, the means of the ratio $R$ decrease with slopes of -0.005 and -0.006 at $28 \mathrm{GHz}$ and $39 \mathrm{GHz}$ in the villa district environment, respectively, and are both -0.002 in the industrial park environment. The high rate of descent (i.e., $|\beta|$ ) in the villa district environment, especially at $39 \mathrm{GHz}$, shows that relatively less foliage scattering can be distinguished with increasing TX-RX separation in densely vegetative areas, and smaller wavelengths may result in very significant small-scale fading in the mmWave band. 
The statistics of the delay and angular spreads for the dominant or first-arrival cluster (i.e., the LoS cluster in the LoS setting and the OLoS cluster in the OLoS setting) are extracted and given in Table IV. In comparison with the results over all available clusters provided in Table III, the delay spreads of the dominant cluster for both LoS and OLoS links are smaller than the overall results. Note, however, that the mean ASAs of the first-arrival cluster in the villa district are $16.93^{\circ}$ and $15.90^{\circ}$ at $28 \mathrm{GHz}$ and $39 \mathrm{GHz}$, respectively, and are $21.68^{\circ}$ and $16.55^{\circ}$ in the industrial park environment, all being higher than the corresponding values for the combined results. Hence, it is reasonable to conclude that propagation through foliage will increase the angular spread of the forward dominant cluster, wherein the MPCs are more concentrated in the time domain (i.e., smaller delay spread) compared with other reflected or scattered clusters. This result is consistent with the measurement-based results in the microwave band [45], [46].

\section{CONClusion}

An empirically based analysis of mmWave large-scale and small-scale propagation characteristics in the two vegetated suburban macrocell environments with different types and fractions of vegetation cover has been presented. Based on the empirical results across the $28 \mathrm{GHz}$ and $39 \mathrm{GHz}$ bands, the CI model is utilized to characterize directional and omnidirectional path loss in the OLoS setting, which shows significant attenuation in vegetation relative to the path loss in the LoS setting. In comparison with the ITU-R suburban and 3GPP RMa models, the effect of dense-leaved trees on the omnidirectional path loss is similar to that of buildings. However, they are inapplicable to cellular coverage planning in sparsely vegetative areas. Consequently, considering the knowledge of transceiver siting, beam misalignment, and physical blockages, an azimuth-angle-based large-scale fading characterization has been presented, which is more appropriate for determining the mmWave radio link budget.

Regarding the composite dispersion parameters, propagation through vegetation remarkably increases the RMS delay and angular spreads compared with the reference measurement results for the LoS link. The cluster-level analyses reveal that vegetated suburban areas are not multipath-rich environments because they possess fewer clusters. In addition, forward scattering by obstructing foliage is the dominant propagation mechanism for OLoS links, corresponding to an increase in ASA for the first-arrival cluster containing more compact MPCs in the delay domain. The measurement-based statistics and modeling of the path loss, shadow fading, delay and angular spreads, and cluster-level parameters complement the existing 3GPP model for suburban macrocell scenarios at mmWave bands.

\section{ACKNOWLEDGMENT}

The authors wish to thank the editors and anonymous reviewers for their valuable comments and suggestions. And the authors are also grateful to their colleagues in the School of Information Science and Engineering, Southeast University,
Nanjing, China, for their valuable discussions and support in measurements.

\section{REFERENCES}

[1] D. Soldani and A. Manzalini, "Horizon 2020 and beyond: On the 5G operating system for a true digital society," IEEE Veh. Technol. Mag., vol. 10 , no. 1 , pp. 32-42, Mar. 2015.

[2] M. Shafi et al., "5G: A tutorial overview of standards, trials, challenges, deployment, and practice," IEEE J. Sel. Areas Commun., vol. 35, no. 6, pp. 1201-1221, June 2017.

[3] F. Boccardi et al., "Spectrum pooling in mmWave networks: Opportunities, challenges, and enablers," IEEE Commun. Mag., vol. 54, no. 11, pp. 33-39, Nov. 2016.

[4] T. S. Rappaport et al., "Millimeter wave mobile communications for 5G cellular: It will work!” IEEE Access, vol. 1, pp. 335-349, 2013.

[5] T. S. Rappaport, G. R. MacCartney, M. K. Samimi, and S. Sun, "Wideband millimeter-wave propagation measurements and channel models for future wireless communication system design," IEEE Tran. Commun., vol. 63, no. 9, pp. 3029-3056, Sept. 2015.

[6] J. Ko et al., "Millimeter-wave channel measurements and analysis for statistical spatial channel model in in-building and urban environments at $28 \mathrm{GHz}$,' IEEE Trans. Wireless Commun., vol. 16, no. 9, pp. 58535868, Sept. 2017.

[7] H. Wang, P. Zhang, J. Li, and X. You, "Radio propagation and wireless coverage of LSAA-based 5G millimeter-wave mobile communication systems," China Commun., vol. 16, no. 5, pp. 1-18, May 2019.

[8] P. Zhang, J. Li, H. Wang, and X. You, "Millimeter-wave space-time propagation characteristics in urban macrocell scenarios," in Proc. IEEE Int. Conf. Commun. (ICC), Shanghai, China, May 2019, pp. 1-6.

[9] J. Li, P. Zhang, H. Wang, C. Yu, and W. Hong, "High-efficiency millimeter-wave wideband channel measurement system," in Proc. Eur. Conf. Antennas Propag. (EuCAP), Krakow, Poland, Apr. 2019, pp. 1-5.

[10] P. B. Papazian, C. Gentile, K. A. Remley, J. Senic, and N. Golmie, "A radio channel sounder for mobile millimeter-wave communications: System implementation and measurement assessment," IEEE Trans. Microw. Theory Tech., vol. 64, no. 9, pp. 2924-2932, Sep. 2016.

[11] C. U. Bas et al., "A real-time millimeter-wave phased array MIMO channel sounder," in Proc. IEEE 86th Veh. Technol. Conf. (VTC-Fall), Toronto, ON, Canada, Sep. 2017, pp. 1-6.

[12] X. Zhao et al., "Attenuation by human bodies at 26- and $39.5-\mathrm{GHz}$ millimeter wavebands," IEEE Antennas Wireless Propag. Lett., vol. 16, pp. 1229-1232, 2017.

[13] S. Morgadinho et al., "Time-variant radio channel characterization and modelling of vegetation media at millimeter-wave frequency," IEEE Trans. Antennas Propag., vol. 60, no. 3, pp. 1557-1568, Mar. 2012.

[14] T. S. Rappaport and S. Deng, "73 GHz wideband millimeter-wave foliage and ground reflection measurements and models," in Proc. IEEE Int. Conf. Commun. Workshop (ICCW), London, UK, June 2015, pp. $1238-1243$.

[15] C. Zhang, X. Yin, X. Cai, and Z. Yu, "Wideband 39 GHz millimeterwave channel measurements under diversified vegetation," in Proc. IEEE Int. Symp. Pers., Indoor Mobile Radio Commun. (PIMRC), Bologna, Italy, Sep. 2018, pp. 1-6.

[16] H. Zhao et al., " $28 \mathrm{GHz}$ millimeter wave cellular communication measurements for reflection and penetration loss in and around buildings in New York city," in Proc. IEEE Int. Conf. Commun. (ICC), Budapest, Hungary, June 2013, pp. 5163-5167.

[17] J. Du, D. Chizhik, R. Feick, G. Castro, M. Rodriguez, and R. A. Valenzuela, "Suburban residential building penetration loss at $28 \mathrm{GHz}$ for fixed wireless access," IEEE Wireless Commun. Lett., vol. 7, no. 6, pp. 890-893, Dec. 2018.

[18] ITU, "Propagation data and prediction methods for the planning of short-range outdoor radiocommunication systems and radio local area networks in the frequency range $300 \mathrm{MHz}$ to $100 \mathrm{GHz}$,' ITU-R, Rec., Tech. Rep. P.1411-9, June 2017.

[19] I. Rodriguez, E. P. L. Almeida, R. Abreu, M. Lauridsen, A. Loureiro, and P. Mogensen, "Analysis and comparison of $24 \mathrm{GHz}$ cmWave radio propagation in urban and suburban scenarios," in Proc. IEEE Wireless Commun. Netw. Conf. (WCNC), Doha, Qatar, Apr. 2016, pp. 1-7.

[20] C. U. Bas et al., " $28 \mathrm{GHz}$ microcell measurement campaign for residential environment," in Proc. IEEE Global Commun. Conf. (GLOBECOM), Singapore, Singapore, Dec. 2017, pp. 1-6.

[21] Y. Zhang et al., "28-GHz channel measurements and modeling for suburban environments," in Proc. IEEE Int. Conf. Commun. (ICC), Kansas City, MO, USA, May 2018, pp. 1-6. 
[22] —. (2017, Nov.) 28-GHz channel measurements and modeling for suburban environments. [Online]. Available: https://docs.lib.purdue.edu/ cgi/viewcontent.cgi?article $=1488 \&$ context $=$ ecetr

[23] M. Ding and D. Lopez-Perez, "Performance impact of base station antenna heights in dense cellular networks," IEEE Trans. Wireless Commun., vol. 16, no. 12, pp. 8147-8161, Dec. 2017.

[24] Z. Zhong, C. Li, J. Zhao, and X. Zhang, "Height-dependent path loss model and large-scale characteristics analysis of $28 \mathrm{GHz}$ and 38.6 $\mathrm{GHz}$ in urban micro scenarios," in Proc. Eur. Conf. Antennas Propag. (EuCAP), Paris, France, Mar. 2017, pp. 1818-1822.

[25] V. Monebhurrun, "Numerical and experimental investigations of base station antenna height on cellular network coverage," IEEE Trans. Antennas Propag., vol. 66, no. 12, pp. 6563-6569, Dec. 2018.

[26] M. O. Al-Nuaimi and R. B. L. Stephens, "Measurements and prediction model optimisation for signal attenuation in vegetation media at centimetre wave frequencies," IEE Proc.-Microw. Antennas Propag., vol. 145, no. 3, pp. 201-206, June 1998.

[27] N. C. Rogers et al., "A generic model of 1-60 GHz radio propagation through vegetation - Final Report," UK Radiocommun. Agency, Tech. Rep. QINETIQ/KI/COM/CR020196/1.0, May 2002.

[28] I. Rodriguez, R. Abreu, E. P. L. Almeida, M. Lauridsen, A. Loureiro, and P. Mogensen, " $24 \mathrm{GHz}$ cmWave radio propagation through vegetation: Suburban tree clutter attenuation," in Proc. Eur. Conf. Antennas Propag. (EuCAP), Davos, Switzerland, Apr. 2016, pp. 1-5.

[29] J. Ko et al., "28 GHz millimeter-wave measurements and models for signal attenuation in vegetated areas," in Proc. Eur. Conf. Antennas Propag. (EuCAP), Paris, France, Mar. 2017, pp. 1808-1812.

[30] C. U. Bas et al., " $28 \mathrm{GHz}$ foliage propagation channel measurements," in Proc. IEEE Global Commun. Conf. (GLOBECOM), Abu Dhabi, United Arab Emirates, Dec. 2018, pp. 1-6.

[31] N. R. Leonor, T. R. Fernandes, M. G. Sanchez, and R. F. S. Caldeirinha, "A 3-D model for millimeter-wave propagation through vegetation media using ray-tracing," IEEE Trans. Antennas Propag., vol. 67, no. 6, pp. 4313-4318, June 2019.

[32] ITU, "Attenuation in vegetation," ITU-R, Rec., Tech. Rep. P.833-9, Sept. 2016.

[33] M. Celidonio, E. Fionda, M. Vaser, and E. Restuccia, "NLOS mmWave propagation measurements through vegetation in urban area: a case study," in Proc. AEIT Int. Annu. Conf., Bari, Italy, Oct. 2018, pp. 16.

[34] A. M. Al-Samman and T. A. Rahman, " $37 \mathrm{GHz}$ wideband millimeterwave radio propagation measurement in foliage environment," in Proc. Int. Symp. Antennas Propag. (ISAP), Busan, Korea (South), Oct. 2018, pp. $1-2$.

[35] 3GPP, "Study on channel model for frequency from 0.5 to $100 \mathrm{GHz}$," 3GPP, Tech. Rep. 38.901 (V15.0.0), June 2018.

[36] ITU, "Multipath propagation and parameterization of its characteristics," ITU-R, Rec., Tech. Rep. P.1407-6, June 2017.

[37] S. Sun, T. A. Thomas, T. S. Rappaport, H. Nguyen, I. Z. Kovacs, and I. Rodriguez, "Path loss, shadow fading, and line-of-sight probability models for 5G urban macro-cellular scenarios," in Proc. IEEE Globecom Workshops (GC Wkshps), San Diego, CA, USA, Dec. 2015, pp. 1-7.

[38] N. Kita, W. Yamada, and A. Sato, "Path loss prediction model for the overrooftop propagation environment of microwave band in suburban areas," Electronics Commun. Japan (Part I: Commun.), vol. 90, pp. 1324, Jan. 2007.

[39] J. G. Andrews, T. Bai, M. N. Kulkarni, A. Alkhateeb, A. K. Gupta, and R. W. Heath, "Modeling and analyzing millimeter wave cellular systems," IEEE Tran. Commun., vol. 65, no. 1, pp. 403-430, Jan. 2017.

[40] W. Lu and M. D. Renzo, "Stochastic geometry modeling of cellular networks: Analysis, simulation and experimental validation," in Proc. ACM Int. Conf. Modeling, Anal. Simulation Wireless Mobile Syst., 2015, pp. $179-188$.

[41] J. Holis and P. Pechac, "Elevation dependent shadowing model for mobile communications via high altitude platforms in built-up areas," IEEE Trans. Antennas Propag., vol. 56, no. 4, pp. 1078-1084, Apr. 2008.

[42] P. Zhang, H. Wang, Y. Zhou, X. Sun, and H. Wang, "An improved three-dimensional auto-clustering algorithm for indoor millimeter-wave multipath propagation analysis," in Proc. IEEE/CIC Int. Conf. Commun. China (ICCC), Qingdao, China, Oct. 2017, pp. 1-6.

[43] X. Yin, C. Ling, and M. Kim, "Experimental multipath-cluster characteristics of $28-\mathrm{GHz}$ propagation channel," IEEE Access, vol. 3, pp. $3138-3150,2015$.

[44] B. H. Fleury, "First- and second-order characterization of direction dispersion and space selectivity in the radio channel," IEEE Trans. Inf. Theory, vol. 46, no. 6, pp. 2027-2044, Sep. 2000.
[45] M. Ghoraishi, J. Takada, and T. Imai, "Analysis of mobile radio wave dispersion through vegetation," in Proc. Eur. Conf. Antennas Propag. (EuCAP), Prague, Czech Republic, Mar. 2012, pp. 3366-3370.

[46] M. Ghoraishi, J. Takada, C. Phakasoum, T. Imai, and K. Kitao, "Azimuth and delay dispersion of mobile radio wave propagation through vegetation," in Proc. Eur. Conf. Antennas Propag. (EuCAP), Barcelona, Spain, Apr. 2010, pp. 1-4.

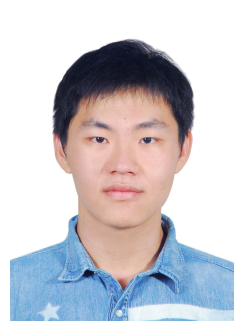

Peize Zhang (S'18) received the B.S. degree (Hons.) from Beijing University of Posts and Telecommunications, Beijing, China, in 2015, and the M.S. degree (Hons.) from China Academy of Telecommunications Technology, Beijing, China, in 2018, both in Electrical Engineering. He is currently pursuing the $\mathrm{Ph} . \mathrm{D}$. degree at the State Key Laboratory of Millimeter Waves, Southeast University, Nanjing, China. His current research interests include millimeter-wave radio propagation measurements, channel modeling, and millimeter-wave wireless network design.

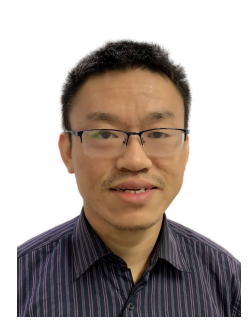

Bensheng Yang (S'17) received the B.S. degree from Northeastern University at Qinhuangdao, China, in 2013, and the M.S. degree from Xidian University, Xi'an, China, in 2016. He is currently pursuing the Ph.D. degree at the State Key Laboratory of Millimeter Waves, Southeast University, Nanjing, China.

From 2016 to 2017, he was an Engineer with Huawei Technologies Co., Ltd., Nanjing, China. His current research interests include electromagnetic vector antenna design, radio propagation measurements, and signal processing.

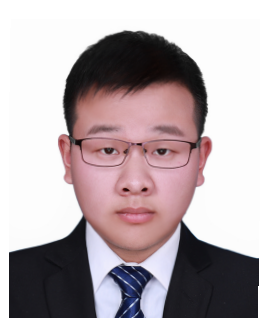

Cheng Yi received the B.S. degree in information engineering from Southeast University, Nanjing, China, in 2016. He is currently working toward the M.S. at the State Key Laboratory of Millimeter Waves, Southeast University.

His main research interests include flexible millimeter-wave channel sounder design and channel modeling. 


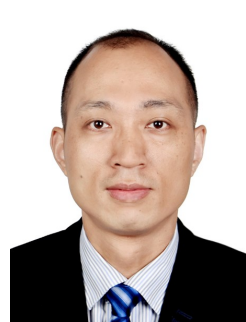

Haiming Wang (M'08) was born in 1975. He received the B.S., M.S. and Ph.D. degrees in Electrical Engineering from Southeast University, Nanjing, China, in 1999, 2002 and 2009, respectively.

He joined the State Key Laboratory of Millimeter Waves, Southeast University, in April 2002. Now he is a professor. In 2008, he was a short-term visiting scholar at the Blekinge Institute of Technology, Sweden. His current research interests include millimeter-wave wireless mobile communications, millimeter-wave radar and imaging, radio propagation measurement and channel modelling, multi-band and broadband antennas and arrays.

He has authored and co-authored over 50 technical publications in IEEE Trans. Antennas Propag., IEEE Antennas Wireless Propag. Lett. and other peer-reviewed academic journals. He has authored and co-authored over more than 60 patents and 30 patents have been granted. Dr. Wang received the firstclass Science and Technology Progress Award of Jiangsu Province of China in 2009. He served as the vice chair of IEEE 802.11aj task group from September 2012 to July 2018. He was awarded for contributing to the development of IEEE 802.11aj by the IEEE-SA in July 2018.

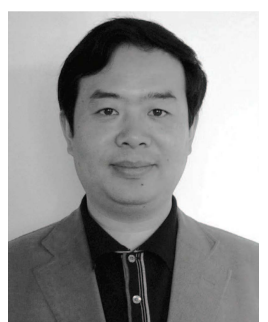

Xiaohu You (F'11) was born in August 25, 1962. $\mathrm{He}$ received his Master and Ph.D. Degrees from Southeast University, Nanjing, China, in Electrical Engineering in 1985 and 1988, respectively. Since 1990, he has been working with National Mobile Communications Research Laboratory at Southeast University, where he holds the rank of director and professor. His research interests include mobile communication systems, signal processing and its applications. He has contributed over 100 IEEE journal papers and 2 books in the areas of adaptive signal processing, neural networks and their applications to communication systems. From 1999 to 2002, he was the Principal Expert of the C3G Project, responsible for organizing China's 3G Mobile Communications R\&D Activities. From 2001-2006, he was the Principal Expert of the China National 863 Beyond 3G FuTURE Project. Since 2013, he has been the Principal Investigator of China National 863 5G Project.

Professor You served as the general chairs of IEEE WCNC 2013, IEEE VTC 2016 Spring and IEEE ICC 2019. Now he is Secretary General of the FuTURE Forum, vice Chair of China IMT-2020 Promotion Group, vice Chair of China National Mega Project on New Generation Mobile Network. He was the recipient of the National First Class Invention Prize in 2011, and he was selected as IEEE Fellow in same year due to his contributions to development of mobile communications in China. 